\title{
EL ROL DEL PSICÓLOGO COMUNITARIO DESDE UNA COMUNIDAD EN LA ARAUCANÍA: UNA EXPERIENCIA DE APRENDIZAJE-SERVICIO
}

\author{
THE ROLE OF THE COMMUNITY PSYCHOLOGIST FROM A COMMUNITY \\ IN ARAUCANIA: A SERVICE-LEARNING EXPERIENCE
}

\author{
Saul Miranda Ramos*, Sebastián Huenupil González**, José Friz Muñoz** \\ * Universidad Autónoma de Barcelona (España), **Universidad Católica de Temuco (Chile)
}

\begin{abstract}
Correspondencia: Saul Miranda Ramos Correo: saulmirandaramos@gmail.com Recibido: 31-03-2020 Aceptado:25/05/2020 DOI: 10.17398/0213-9529.40.1.87
\end{abstract}

\section{RESUMEN}

El aprendizaje en el contexto comunitario ofrece a los estudiantes nuevos estímulos a la hora de adquirir un conocimiento favoreciendo a su vez la vinculación universidad y sociedad. Esta experiencia parte de la realización del Proyecto de Tesis para la obtención del título de Diplomado Internacional en Psicología Comunitaria: Metodologías para la acción social en salud, educación y organizaciones, en la Universidad Católica de Temuco (Chile), ofreciéndose como una oportunidad ideal para estudiar el entorno real, en este caso la comunidad en la Araucanía, y proponer alternativas desde la perspectiva de la Psicología Comunitaria (PC). Los resultados de la experiencia muestran que la realidad del espacio comunitario abre un abanico de elementos que el PC debe considerar para su praxis y evidenciaron prácticas verticales, que permitieron generar reflexiones en torno al planteamiento del problema. Como conclusiones, el PC fue observado como un facilitador de recursos intangibles.

Palabras clave: aprendizaje-servicio, asistencialismo, Psicología comunitaria, Políticas Públicas, verticalidad.

\begin{abstract}
Learning in the community context offers students new stimuli in the acquisition of knowledge, which in turn promotes the link between university and society. This experience is based on the Thesis Project for the International Diploma in Community Psychology: Methodologies for social action in health, education and organizations, at the Catholic University of Temuco (Chile), offering an ideal opportunity to study the real environment, in this case the community in Araucania, and propose alternatives from the perspective of Community Psychology (CP). The results of the experience show that the reality of the community space opens a range of elements that the CP must consider for its praxis and evidenced vertical practices, which allowed to generate reflections around the approach of the problem. As conclusions, the PC was observed as a facilitator of intangible resources.
\end{abstract}

Keys words: Service-learning, assistance, Community Psychology, Public Policies, verticality.

Conflicto de intereses / Conflicts of Interest: Los autores no declaran conflicto de intereses.

Sección / Section: El Aprendizaje Servicio (ApS) en la sociedad actual

Editor de Sección / Edited by:,Ana López Medialdea - Nieves Martin Bermúdez / Universidad de Extremadura, España

Campo Abierto, v. 40, n. 1, p. 87-102, 2021 


\section{INTRODUCCIÓN}

La Educación Superior se brinda como espacio ideal para la formación de profesionales que desarrollen competencias basadas en el pensamiento crítico en busca de una transformación social. Es por ello, que se plantea la importancia de buscar nuevos planteamientos que cuestionen las propuestas pedagógicas sobre todo en las disciplinas sociales, en las que el aprendizaje se desarrolle más allá del aula universitaria. El aprendizaje en el contexto comunitario ofrece a los estudiantes nuevos estímulos a la hora de adquirir un conocimiento que van más allá de la adquisición de contenidos teóricos. Es decir, favorece la vinculación universidad y sociedad.

El punto de partida para el desarrollo de esta experiencia fue la realización del Proyecto de Tesis para la obtención del título de Diplomado Internacional en Psicología Comunitaria: Metodologías para la acción social en salud, educación y organizaciones, en la universidad Católica de Temuco (Chile). El desarrollo de este aprendizaje se ofrece como una oportunidad ideal para conocer el contexto concreto para estudiar el entorno real, en este caso la comunidad en la Araucanía, y proponer alternativas desde la perspectiva de la Psicología Comunitaria.

Este enfoque al aprendizaje se enmarca en lo que define proyecto de aprendizaje-servicio ya que el proceso de aprendizaje se realiza sobre entornos reales y los describe de la siguiente manera: "una propuesta educativa que combina procesos de aprendizaje y de servicio a la comunidad en un solo proyecto bien articulado en el que los participantes se forman al trabajar sobre necesidades reales del entorno con el objetivo de mejorarlo" (Puig, Batlle, Bosch, y Palos, 2007, p. 20)

Por otra parte, Tapia (2018, p. 19), concreta los beneficios que arroja este tipo de proyectos centrándose en los siguientes aspectos:

- Fortalecen la calidad educativa ya que para solventar problemáticas concretas hay que saber más allá de los contenidos teóricos o realizar una evaluación, y porque en el terreno se aprenden conocimientos, se adquieren competencias y habilidades que no pueden encontrarse en los manuales.

- Educan para la ciudadanía ya que van más allá del diagnóstico de necesidades, avanzan en el diseño y desarrollo de acciones transformadoras de la realidad.

- Plantean prácticas inclusivas y contribuyen a la participación activa de proyectos de desarrollo local.

- Permiten la organización de redes entre la institución educativa y la organización de la comunidad, facilitando la tarea docente y posibilitando la creación de soluciones conjuntas a problemas comunes.

Algunas de las formas para combatir esta desigualdad pueden nacer desde ciertas decisiones estatales que buscan dar solución a la corrupción, la inequidad y la injusticia social (García, Priego y Rebollo, 2018) desde espacios lejanos a los territorios. Dentro de este campo, se mueve el trabajo del Psicólogo/a Comunitario/a (PC) que busca, desde el hacer común, lograr la emancipación y construir nuevas significantes transformadoras. Más aún, de sus postulados se desprende ideas como el fortalecimiento comunitario, en el cual las comunidades dirigen sus acciones en beneficio propio, detectan sus problemáticas y desarrollan acciones para superarlas (Montero, 2003) y es donde surge la sinergia directa con los procesos de Aprendizaje-Servicio.

Así también, en la Educación Popular, a través del pensamiento de Freire, el PC puede realizar diversas intervenciones con instrumentos que permitan -desde el mundo del saber e historias de vida de los sujetos populares- forjar fortalecimiento territorial, donde las personas se transformen en actores sociales activos (Restrepo y González, 2018). Desde estas ideas se 
extrae una crítica hacia el modelo de mercado de (re)producción del conocimiento, en la cual el educador es una persona que amaestra y aliena al educando (educación bancaria), disociado de la realidad del territorio, incurriendo en prácticas asistenciales y lo priva de su libertad creadora de conocimiento y cultura (Rigal, citado por García, 2011).

Estas prácticas voluntarias de carácter participativas tendrían como finalidad permitirle al PC buscar la transformación de los territorios, para avanzar en la liberación de las personas, desde, para y con ellas de manera consciente, en un trabajo de desarrollo y creación cultural desde los saberes populares.

Igualmente, desde la Psicología de la liberación por Martín-Baró se analizan las actitudes del agente profesional a través de una crítica de la ideología, actitudes comprendidas por el autor como la forma en que el sujeto academizado revela las visiones de su mundo y el cómo estas visiones implican una forma de enfrentar lo cotidiano, que generaría dominación (Martín-Baró, 2017). Por ende, Martín-Baró plantea acercar del rol de las/os psicólogas/os una postura más comprometida con lo social y lo político, en un esfuerzo de preocuparse por los problemas sociales de comunidades oprimidas (Martín-Baró, 1990).

En el mismo sentido, el trabajo desde el hacer común también se refleja en el pensamiento de Enrique Dussel, que nace desde sus estudios de la cultura judeo-cristiana que lo llevó a pensar una filosofía distinta, desde abajo, con conocimiento y praxis representativa de la realidad Latinoamericana (Leones, 2018). En este sentido, el hacer común del PC podría brotar desde los pueblos Latinos, incubando pensadores que no repliquen los modelos centralistas/universalistas; modelos desde los cuales se construirían la opresión de clases, la exclusión de países tercermundistas y todo tipo de configuración social alejado de un centro de poder (Dussel, 2017).

En Chile, este quehacer puede ser evidenciado en experiencias de fortalecimiento, como se demuestra en una investigación sobre modelos de abordaje de la violencia contra las mujeres en región de La Araucanía, donde se buscó generar propuestas de manera participativa de tal modo que se pueda ayudar a generar políticas sociales pertinentes para el contexto regional (Ketterer et al., 2017). Igualmente, a modo de ejemplo, esta forma de abordaje se puede observar en el trabajo de Eduardo Almeida, quien realizó un trabajo de cuarenta años con la comunidad de San Miguel Tzinacapan, México, en un proceso de interacción conjunta en búsqueda de la horizontalidad entre indígenas y profesionales urbanos (Almeida y Sánchez, 2014).

Se puede desprender de lo anterior que el ideal del rol del PC se relaciona con un trabajo de facilitación y formación, desde una guía horizontal que promueve la deconstrucción de ciertas premisas opresoras para facilitar la movilización de la conciencia en las personas.

\section{La problemática del rol del Psicólogo Comunitario y Políticas Públicas}

La psicología comunitaria es concebida como "un campo de estudio de la relación entre sistemas sociales entendidos como comunidades, el comportamiento humano y su aplicación interventiva a la prevención de los problemas psicosociales y el desarrollo humano integral" (Sánchez, citado en Arias, 2015, p.1). El psicólogo/a comunitario/a cumple un rol de un experto no directivo y facilitador consultivo, rol que, desde las políticas públicas (PP) en Chile, a pesar de su intento por la democracia participativa en los últimos años, aún recae en labores que pueden ser asistencialistas y verticales. 
En relación a lo anterior, ocurrirían ciertas tensiones entre PC y los lineamientos institucionales que limitarían la relación entre la praxis desde lo público y las bases epistemológicas de lo comunitario, o como lo denomina Shinn (citada por Olivares, Winkler, Reyes, Berroeta y Montero, 2018) determina que los y las psicólogas/os comunitarias/os "bailen con un monstruo", o que tengan que sortear ciertas situaciones que amenacen su labor ética/política. Continuando, estas tensiones se logran evidenciar en un estudio realizado por Reyes, Olivares, Berroeta y Winkler (2015), que abordó las ofertas de programas públicos vigentes en el contexto gubernamental Chileno. El estudio demostró que, de 101 programas/proyectos, sólo 9 cumplieron con criterios para ser considerados "comunitarios"; aun así, la mayoría de los proyectos declararon en sus bases técnicas algunas aplicaciones propias de la Psicología Comunitaria. Además de esto, y según lo indicado por Carrasco, Cerda, Baltar, Zamora y Castillo (2016), existiría cierto desacuerdo entre los intereses que surgen desde el actuar de los/as psicólogos/as comunitarios/as y el obrar desde la institucionalidad bajo planificaciones generadas dentro de la misma. En consecuencia, a pesar de evidenciar cierto aumento de proyectos de intervención comunitaria, es posible inferir que no siempre se logra trabajar desde las características éticas/políticas que sustentan este campo, ya que se aprecian tensiones -desde la institucionalidad del estado y los intereses del PC- al momento de generar espacios de participación efectiva.

En efecto, la labor comunitaria desde PP ha tenido que depender de algunas prácticas tecno-científicas normalizadoras y hegemónicas que se traducen en intervenciones del tipo asistencialistas (Reyes et al, 2015). Estas intervenciones tienden a mantener el "status quo", lo que produciría diversas interferencias que bloquean el alcance emancipador de la Psicología Comunitaria, que mercantilizarían lo común y disminuirían el poder popular para negociar y cuestionar las relaciones que se reproducen en los espacios comunitarios. Esta forma de hacer desde la política social parecería moldear al sujeto desde la carencia, permaneciendo en una condición inhabilitante para dar respuesta a sus propias necesidades e intereses.

Igualmente, desde algunas PP existirían algunas prácticas verticales asociados con abordajes de intervención de modalidad "social-dirigida" (Malluk y D'Luyz, 2016) la cual se caracteriza por ser planificada, ejecutada y evaluada por un equipo técnico/profesional de manera externa. Un ejemplo de prácticas verticales se puede observar en la asignación de proyectos gubernamentales que limitarían el ejercicio de participación efectiva de las personas pertenecientes a los territorios, lo que se traduce en un rol cooperativo de las comunidades en los proyectos que son implantados de forma vertical por parte del Estado (Olguín, Urzúa, Vargas y Vidal, 2016). Esto permite hipotetizar que las comunidades no tendrían mayor incidencia en las decisiones sobre la asignación de recursos públicos y el uso de estos, lo que se traduciría en intervenciones de carácter consultivo y de baja capacidad transformadora.

En resumen, lo planteado indica que, a pesar de que la inclusión histórica de la Psicología Comunitaria en políticas públicas Chilenas ha permitido su consolidación como campo profesional, la práctica en este ámbito ha sido coartada ciertamente de sus principios epistemológicos (Alfaro y Martín, 2015) y quedaría supeditada a una operatividad más vertical y asistencial.

La reflexión sobre la práctica funciona como un medio para estimular el aprendizaje por medio de interacción permanente entre el pensamiento y la acción, permitiendo criticar, reestructurar y planificar aplicaciones para acciones futuras (Posadas y Godino, 2017). Además, permite potenciar el aprendizaje significativo, ya que aquellos sujetos que ponen atención en la forma en que generan sus acciones pueden plantear estrategias propias y valorar de mejor forma la praxis (Matos, 2006). 
Si bien el aprendizaje-servicio es una metodología educativa, una herramienta pedagógica, se puede valorar también desde otras miradas. Más allá de su identidad educativa, el ApS puede ser considerado también como herramienta de desarrollo comunitario, de cohesión de la comunidad (Batlle, 2013, p.5).

Al extrapolar estas nociones con el rol del PC, se logra comprender que la reflexión sobre su labor en las comunidades fomenta un aprendizaje continuo, que se traduce en construcciones de acción estratégicas. Estas construcciones pueden transformar algunas prácticas limitantes del accionar del profesional.

\section{METODOLOGÍA}

Como se anunciaba en líneas anteriores, esta experiencia se basa en la metodología de la Investigación Acción Participativa de corte cualitativa, que permite estudiar una comunidad desde la proposición de acciones con respeto de los saberes territoriales. Los participantes fueron 9 miembros de la Junta de Vecinos N³ del sector "El Alto" de la Comuna de Pitrufquén, en la Región de la Araucanía, Chile. El procedimiento utilizado corresponde a la Animación Sociocultural; partiendo por la toma de contacto, por medio de un informante clave (Presidenta de la Junta de Vecinos, Amelia Riquelme) y la vinculación con los vecinos de la Junta de Vecinos en actividades y reuniones ordinarias. Posteriormente, se llevó a cabo un levantamiento de necesidades propuesto desde la generación de un grupo operativo con los participantes, los cuales trabajaron conjuntamente en el barrio.

En este sentido, además de los objetivos propios del aprendizaje, la experiencia desarrollada planteaba, a su vez, los siguientes objetivos de investigación:

\section{OBJETIVO GENERAL.}

Analizar el rol del Psicólogo comunitario en un proceso de autoestudio participativo en la Junta de Vecinos (JJVV) del Sector el Alto de la Comuna de Pitrufquén en la Región de la Araucanía, Chile, para la elaboración de nuevas significantes transformadoras.

\section{Objetivos Específicos.}

1. Auto-observar el rol del Psicólogo Comunitario en los procesos del Auto-estudio participativo en el sector El Alto de la comuna de Pitrufquén, para la generación de nuevas significantes transformadoras.

2. Fomentar el vínculo positivo entre los agentes externos y la comunidad del Sector el Alto de la Comuna de Pitrufquén que facilite la realización de un trabajo comunitario, a través de la participación en los espacios de interés para los miembros del sector.

3. Generar un levantamiento de necesidades participativo desde la Junta de Vecinos del Sector el Alto de la Comuna de Pitrufquén, para la construcción de nuevas significantes transformadoras.

Para el desarrollo de estos objetivos y sistematizar la experiencia, los instrumentos utilizados fueron la observación participante, compuesta por una tabla de observación de acciones y diálogos para contemplar la realidad comunitaria. También, se utilizó una entrevista semiestructurada para explorar elementos relacionados con la historia de las personas, el barrio y sus costumbres; fue utilizada preferencialmente ya que esta modalidad facilita el desarrollo del vínculo a partir del diálogo. Por último, para llevar un registro de las propias labores, los tesistas utilizaron un Diario de Campo; dividido en tres ámbitos, a saber: 1) descripción de observaciones, 2) descripción de cogniciones y 3) descripción de emociones. 
El análisis de datos fue realizado desde la Teoría Fundamentada (Bénard, 2016), debido al interés de generar nuevas teorías desde los datos emergentes. Para este proceso se utilizó el software Atlas.ti (versión 7.5). Para el uso del resguardo ético se dio a firmar un consentimiento informado a los participantes.

Por último, se aplicaron a las técnicas de recolección de datos como consistencia interna la validación. La credibilidad se realizó a través de la devolución de resultados a la comunidad y la transferibilidad a partir de la descripción detallada del contexto donde se efectuó la investigación, que es la comuna de Pitrufquén.

\section{RESULTADOS}

Los hallazgos encontrados en la comunidad fueron observados dentro de varios hitos.

Primero con la toma de contacto que fue efectuada a través de un informante clave (Presidenta de la JJVV), que permitió indagar en diferentes campos que componen la comunidad, los cuales se relacionan con la salud comunitaria, la educación, algunos elementos culturales, la democracia y las redes, todos observados en las reuniones ordinarias de la JJVV.

Más adelante, en el proceso de levantamiento de necesidades se determinó, de forma conjunta, recopilar información sobre las condiciones de las personas mayores y/o con discapacidad dentro del barrio a través de una encuesta creada por los participantes en las reuniones de trabajo. En esta instancia, debido al clima de la zona, se decidió utilizar los hogares de los miembros del grupo como lugar de reuniones, donde se pudo observar la apertura de un elemento destacable, la "once comunitaria", que abrió la posibilidad de que cada participante llevará una donación que era compartida por el grupo mientras se realizaba el trabajo.

Los resultados de la encuesta fueron sistematizados conjuntamente. Más tarde, el trabajo realizado fue valorado por los mismos participantes, quienes destacaron esta instancia como una oportunidad de aprendizaje y apertura a la cohesión grupal. Finalmente, todos los elementos observados se pudieron englobar en dos categorías, los componentes influyentes (divididos en amenazas y recursos) y las competencias del PC, que sumados con los campos de acción participativa observados en las reuniones de la JJVV, buscan dar cuenta de los ámbitos relacionados con el rol del PC identificados en la realidad comunitaria del sector el alto, de la comuna de Pitrufquén.

\section{El rol del psicólogo comunitario en la realidad compleja}

La realidad comunitaria observada proyecta ciertos ámbitos relacionados a la praxis del PC. A continuación, estos ámbitos se entregan de manera sistematizada. (figura 1)

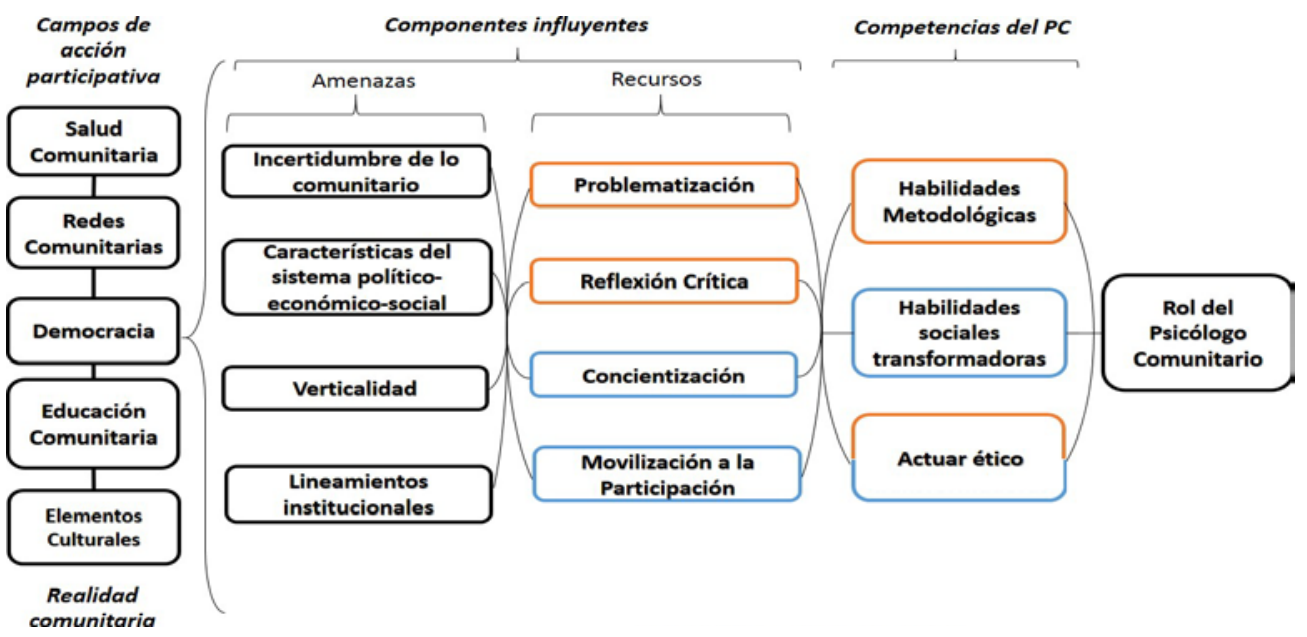

Figura 1. Modelo de ámbitos influyentes en el rol del psicólogo/a comunitario 


\section{Campos de acción participativos.}

a) La Salud comunitaria: Relacionada con el bienestar biopsicosocial de los miembros de la comunidad, donde el PC puede intervenir desde la promoción de hábitos de vida saludable y prevención de enfermedades. En la praxis, la salud, fue abordada a partir de la aplicación de encuestas enfocadas a personas mayores, cuyos resultados apuntaron a la necesidad de involucrar a expertos externos en salud dentro de la comunidad. La definición anterior puede ser contrastada con el siguiente relato de un vecino del barrio: "Nos gustaría que nos atendieran médicos especialistas para tener una mejor calidad de vida en nuestra sede (una o dos veces al mes)." (Nota de campo). Lo expuesto permite observar una inquietud respecto a una necesidad sentida en el barrio, que involucra a un global del sector y que puede ser atendida según las gestiones que se realicen para contactar profesionales externos.

b) Redes comunitarias: Asociado a las relaciones dentro de la comunidad y/u otros sistemas como redes de apoyo. En la comunidad, se logró evidenciar que, por lo general, estas redes se mueven bajo la lógica de intercambio de intereses, donde el/la PC, al igual que otros miembros externos, pueden servir como facilitador/a de recursos materiales 0 intangibles. Esto se puede visualizar en el siguiente registro: "Se habla sobre patrulla comunitaria. Se establecen redes de apoyo con carabineros para capacitaciones y charlas en la junta (...). El sub-oficial entrega los teléfonos de emergencia de la guardia 24-7, patrullas comunitarias y de la sargento" (Nota de campo). Lo anterior presenta una red que se gesta gracias a la vinculación de una institución del estado y la JJVV que puede avanzar en responder a diversas inquietudes y necesidades del entorno.

c) Democracia: Implica las formas de participación, validación y valoración de las personas dentro de la comunidad. También se relaciona con la toma de decisiones conjuntas, que buscan dar respuesta a situaciones o necesidades de un sector determinado. Esto se observa en la siguiente nota de campo: "Pdta propone: utilizar dinero (del evento de 18 de septiembre) para comprar una mejor estufa y cocina de leña para calefaccionar la sede $y$ poder realizar talleres de repostería. Los/as vecinos/as presentes (15 aprox) aprueban la propuesta". (Nota de campo). Lo registrado evidencia un diálogo de carácter horizontal, en el cual las/los vecinos/as pueden dialogar respecto a una idea que surge desde una dirigente comunitaria, lo cual a la vez demuestra una apertura al diálogo para la toma de decisiones.

d) Educación comunitaria: Asociada con las formas de transmitir conocimiento dentro del espacio comunitario de forma participativa. En este aspecto, el PC puede integrar dinámicas que permitan educar desde el hacer, (co)construyendo conocimientos que cada participante pueda desarrollar. Los espacios de análisis conjunto del territorio comunitario facilitan el compartir conocimientos, en relación a lo común para las personas del grupo, como también, para los investigadores.

e) Los elementos culturales: Referido a aquellas costumbres integradas desde el exterior o aquellos hábitos creados dentro de la comunidad. Se puede comprender que existen elementos idiosincráticos que forman parte de una costumbre dentro del territorio, que permite dar cuenta de formas de compartir y que permiten abrir los diálogos. 


\section{Los componentes influyentes en el rol del PC.}

\section{Amenazas.}

a) La incertidumbre de lo comunitario: Referido a aquellas situaciones esperadas o inesperadas que puedan suceder en la realidad comunitaria: "Trabajar con gente es difícil, he visto gente golpeándose, vecinos que se odian", (Observación participante). Así también, la incertidumbre se presentó como un factor limitante, ya que, debido a situaciones imprevistas, se tuvo que re-agendar actividades pre-programadas. Así se demuestra en la siguiente cita: "Para esta reunión se optó por informar a la gente de una forma más personal a través de invitaciones. El resultado fue que llegó solo una persona", (Nota de campo). Lo anterior constata diversas situaciones que pudieron mermar actividades, como también dan cuenta de la complejidad de algunas realidades barriales que los/as profesionales comunitarios podrían sortear.

b) Las características del sistema político: Se relaciona con algunos comportamientos caracterizados por una individualización y debilitamiento de los lazos sociales que podrían estar relacionados con algunas características del sistema político, que tendrían influencia directa o indirecta en los abordajes del PC en la comunidad. Como por ejemplo, el desinterés por el otro y la baja la participación; términos que se puede asociar a los siguientes registros: "Los vecinos con suerte se saludan, es difícil comunicarse (...). Nosotros venimos de Santiago y se ve difícil." (Observación participante). Se identifican ciertas fragmentaciones sociales en diversos grupos, como son las relaciones entre vecinos/as y entre grupos etarios, además de algunas afecciones a grupos etarios específicos, producto de inadecuaciones en las estructuras del espacio público.

c) La verticalidad: Se asocia con abordajes de intervención propuestas, diseñadas, ejecutadas y evaluadas desde afuera por parte de los/as expertos/as, que dejarían de lado la participación comunitaria y se implementaría un trabajo desde arriba -en el cual se indica a las personas beneficiadas lo que deben hacer, cómo y cuándo-, se silenciaría, por tanto, el saber común representativo de un territorio determinado. En la presente experiencia se logró evidenciar la posibilidad de caer en este tipo de prácticas, como lo demuestran los siguientes registros: "A pesar de nuestros intentos de generar conocimiento autónomo, recaímos en el dogma y en la costumbre", (Nota de campo). De esta forma, en la praxis, se vivenciaron momento en los cuales se obró de forma vertical a pesar de los intentos de trabajar desde un posicionamiento horizontal y participativo, lo que indicaría que existieron momentos donde las personas del grupo cumplieron un rol pasivo.

d) Los lineamientos institucionales: Se asocian con algunos protocolos burocráticos que pueden provenir de espacios estatales o en este caso, de la academia, que generan ciertas exigencias para el PC y que representarían un riesgo para el compromiso con la comunidad. Así se evidenció esto en el grupo de tesistas, referido a la presión académica. Estas circunstancias, sumadas a la capacidad o incapacidad de los sujetos interventores de sortear alternativas, puede terminar por generar ciertos patrones de trabajo situados desde la verticalidad.

\section{Recursos.}

a) La problematización: Implica un cuestionamiento propuesto por el PC hacia una problemática, que da la apertura a una reflexión. Esto se observó durante las reuniones ordinarias dentro de la JJVV $\mathrm{N}^{\circ} 3$ y así lo afirma una de sus integrantes: "¿Cómo podríamos encauzar los diálogos para que se busquen alternativas de diálogo con los y las vecinas que se ven envueltas en las declaraciones polémicas?", (Nota de campo). Esta 
frase constata una oportunidad para abrir reflexiones sobre un problema, en este caso relacionado con la apertura de diálogos, e invita posteriormente al PC a utilizar esto para develar constructos socio-políticos ocultos tras estas realidades.

b) Reflexión crítica: Se asocia con la problematización y tiene que ver con los debates y diversas opiniones que intentan dilucidar los elementos socio-políticos que se encuentren invisibilizados en el sector. Esto se evidenció en el grupo operativo, por ejemplo, en diálogos respecto a situaciones que se relacionan con la perspectiva de género: "hoy se están terminando los estereotipos de la mujer que tiene que llorar y el hombre macho, pero aún falta para llegar a la equidad de género", (Observación participante). Estas reflexiones críticas permitirían al PC generar alternativas, encaminadas a la desideologización, que inviten a la movilización de conciencia, favoreciendo la construcción de un nuevo papel en las personas involucradas.

c) La concientización: Se relaciona con la toma de conciencia sobre propias condiciones que pueden encontrarse alineadas o no integradas, facilitada desde el PC u otro participante de una discusión, por medio de problematizaciones y reflexiones en torno a un tema, así se evidencia en el siguiente registro sobre el machismo en la sociedad chilena: "La señora María explica que cada uno es como quiere ser. Dice que siempre se atrevió a realizar actividades de hombres en el campo. Luego, al problematizar esta realidad de ella, se da cuenta que nadie se lo impidió en su niñez y que esto fue gracias a sus padres, que le permitieron desarrollarse de forma más completa", (Nota de campo). Lo anterior permitiría avanzar en un nuevo entendimiento de sus condiciones territoriales y sociales, pudiendo abrir nuevos diálogos respecto a diversas temáticas afectadas por la inequidad, para así generar consensos y abrir camino a movilizar la conciencia de forma conjunta.

d) La Movilización a la participación: Implica los esfuerzos del PC u otros miembros de la comunidad por la inclusión de nuevas concepciones y aproximaciones que se caractericen por el respeto por los saberes barriales e integrar nuevos participantes activos en las asambleas con la finalidad de fortalecer las bases comunitarias, esfuerzos que pueden ser asociados a los siguientes registros: "La presidenta da la bienvenida a la vecina que llegó de Galvarino Rivero, la invitó a seguir formando parte del espacio y a seguir trabajando juntas." (Observación participante); "Se invita a los vecinos a participar en el análisis de datos y en el trabajo investigativo de observación participante", (Nota de campo). Estos diálogos revelan un interés y acciones concretas que llamarían a la participación de los/las vecinos/as para fortalecer lazos, junto a la intención de compartir roles en las instancias de investigación.

\section{Las competencias del Psicólogo/a Comunitario/a.}

a) Habilidades metodológicas: Implica aquellos recursos que, por formación académica, le permiten al PC desenvolverse dentro de los procesos interventivos en la comunidad. De estos, se destaca principalmente el manejo de conocimiento teórico, metodológico y aplicación de la disciplina, evidenciado principalmente en la capacidad de problematizar sobre la realidad por parte del PC, como la capacidad de levantar procesos de reflexión crítica sobre variados aspectos relacionados con en el barrio. A pesar de esto, durante el proceso se pudieron registrar diversos aciertos y errores en tanto a lo metodológico que se relacionan con la inexperiencia y desconocimiento de los tesistas en el campo de acción, ya que no lograron ejecutar ciertos lineamientos de la manera más adecuada para entregar cierta información a los/as miembros de la comunidad según lo que muestran los siguientes registros: "No supimos explicar la forma de trabajo desde una perspectiva 
comunitaria", (Nota de campo); "La observación participante fue realizada sólo por nosotros (investigadores), probablemente por no saber explicar el propósito de esta", (Nota de campo). A través de lo anterior, es posible visibilizar ciertos recursos que, a pesar de permitir la facilitación de procesos de concientización y reflexión, no determinan que el/la PC esté exento de desarrollar ciertas prácticas que pueden ser catalogadas como asistenciales y/o verticales.

b) Habilidades sociales transformadoras: Identificadas como aquellos elementos que le permitirían al PC validarse en las comunidades y facilitar el camino hacia la transformación social, por medio de un accionar diferente al de "agentes externos expertos" y que se relaciona con las formas de concientización y movilización a la participación. En la praxis esto se observó en los esfuerzos por los tesistas de integrarse e integrar a otros en actividades relacionadas a la JJVV, como se evidencia en el siguiente relato: "Ustedes pudieron haberse excusado, pero siempre han sido parte de las actividades y han estado con la disposición. Ustedes querían una oportunidad y la lograron acá. Este proceso es largo y verlo acá nos motiva junto al trabajo realizado en el grupo operativo", (Nota de campo) y por medio de la concientización en las asambleas: "José comienza a hablar sobre cómo el individualismo ha afectado el sentido de unión de la familia. Dice que durante el cambio de siglo se ha dado una pérdida de unión por los matrimonios o de la emigración de los jóvenes por los estudios", (Nota de Campo). Se desprende de lo anterior un posicionamiento comprometido de los investigadores, que se traduciría en una vinculación de carácter sincera, con propiedad de discurso hacia la posibilidad de compartir ideas y con la intención de aportar a través de la facilitación de los diálogos, como también en dar respuestas a necesidades tanto materiales como de capital humano.

c) Actuar ético: Comprendido como la capacidad del profesional de valorar la otredad a través de la aceptación y el respeto de los saberes de los territorios. Estos saberes están íntimamente relacionados con los conocimientos que han sido (re)producido por las personas y por tanto, los/as profesionales debieran comprender que no hay un solo modo de conocer ni una sola explicación para entender los fenómenos sociales. Durante la experiencia se evidenció un desenvolvimiento por parte de los tesistas con resguardo de lo ético a través de la facilitación de espacios de discusión en los cuales se daba cabida a todas las miradas. Esta postura fue recalcada por parte de los vecinos, como se advierte en la nota a continuación: "Ustedes tienen el bicho de lo comunitario, de ver y aportar en el trabajo. nunca se olviden de las viejas de la JJVV”, (Observación participante). Además, el actuar ético se presentó como un marco guía para definir ciertos obstáculos para el PC, como las prácticas verticales, que incluso pudieron formar parte en, cierto momento, de la práctica en lo comunitario. No obstante, durante el proceso, se lograron diálogos que encauzaron la decisión de generar una encuesta barrial desde, para y con las vecinas/os del grupo operativo. Al igual como fue problematizado en las competencias de habilidades metodológicas, el PC se enfrentaría a escenarios diversos que pudieran inclinarlo/a a caer en prácticas alejadas de los postulados éticos, sin embargo, existen otras situaciones que pudieran permitir discusiones que fomenten la participación y la auto-gestión.

Como síntesis se podría afirmar que los ámbitos relacionados con el rol del PC permitirían captar posibles recursos como posibles amenazas en su labor. Todos estos ámbitos se encuentran interrelacionados en la realidad comunitaria y muestran la complejidad que implica la praxis desde el resguardo de los principios epistemológicos del área. De esta manera, la autoobservación y el análisis constante se transformarían en ejes primordiales para que el/la PC pueda efectuar intervenciones que respeten los valores éticos que lo/a sustentan. 


\section{DISCUSIONES}

En primer lugar, como ya se mencionó con anterioridad, el PC podría intervenir en diferentes campos de acción en la realidad comunitaria. En el campo de la salud comunitaria, desde la experiencia en el barrio, se pudo observar que muchas necesidades apuntaron a gestionar acompañamiento para las personas mayores, junto a campañas de salud de diferente índole, con el fin de abaratar costos y desgaste físico de las personas del sector. Este posicionamiento va en contraposición de una perspectiva de salud comunitaria la cual promueve la participación ciudadana y la autosuficiencia de la comunidad como estrategia para el mejoramiento de la salud, añadiendo el elemento de esfuerzo colaborativo (Balcazar, Montero, Newbrough y Porbén, 2003). Aun así, durante el proceso de intervención, se lograron desarrollar discusiones para avanzar en la búsqueda de necesidades desde, para y con las vecinas, al igual que formas de enfrentamiento que incluyeran a las instituciones estatales para mejorar la calidad de vida de las personas, familias y la comunidad.

Refiriéndose a la democracia, y las Redes comunitarias, como posibles campos de acción para el PC, Maritza Montero (2003) comenta que estos ámbitos formarían unas de las orientaciones principales de la Psicología comunitaria, siendo estos una de las finalidades de esta disciplina. Durante el desarrollo de la intervención, el grupo operativo logró espacios democráticos de carácter participativo en los cuales cada perspectiva era valorada y analizada con respeto para ser abordada en la discusión grupal. De igual forma, se buscó apoyo en instituciones, tanto privadas como estatales, con el fin de encontrar soluciones conjuntas y gestar redes permanentes.

Por su parte, la Educación comunitaria fue evidenciada en la JJVV a través de algunas formas de compartir conocimiento. Tanto al interior de la JJVV como del grupo operativo se compartían experiencias y formas de percibir las situaciones por medio de diálogos que permitían la construcción de un posicionamiento grupal para enfrentar la diversidad de problemáticas locales. Lo anterior se relaciona íntimamente con lo que explicó Gloria Graterol (2010), durante un Congreso Iberoamericano de Educación realizado en Buenos Aires, Argentina: Lo común que se hace comunitario se muestra entre realidades complejas dentro de la cotidianidad de las personas que comparten alguna cosa que pertenece a todos. La educación comunitaria, persigue la función social que pretende establecer puentes entre lo social y lo formal. La educación comunitaria tiene entre sus complejas tareas, el reto de tratar de estudiar, entender y retroalimentar la universalidad y pluralidad de las comunidades y sus diversas culturas. (p.2)

Es así como las formas de educación observadas en la JJVV, permitirían el avance de las comunidades en ampliar las formas de participación democrática a través de la toma de decisión conjunta y la co-educación lo que, en el grupo operativo, fue la forma de trabajo que se llevó a cabo.

Por último, los elementos culturales, a través de la visión de Marconi (citado por Alfaro y Barroeta, 2007), son comprendidos desde el relativismo cultural de la antropología social, la cual nos indica que las personas desarrollan sus juicios y comportamientos en base a la experiencia y que cada experiencia es interpretada según los procesos de endoculturación. Estas nociones se apreciaron en el barrio "El Alto", donde es probable que los juicios emergentes en las dinámicas comunitarias respondieran a patrones históricos de comportamiento, así también, la once comunitaria se entendería como una herencia de la idiosincrasia Chilena o costumbre que se practica de manera habitual hasta el día de hoy. 
En segundo lugar, existirían algunos componentes influyentes como amenazas en el rol del PC, siendo uno de estos componentes la incertidumbre de lo comunitario, que desde el pensamiento de Edgar Morin (citado en García, 2017) se relaciona con lo impredecible y lo incierto, influyendo en aspectos relacionados con el conocimiento humano, la lógica racional y la mente. En ese sentido el conocimiento implica acciones, decisiones y apuestas dentro de una realidad compleja llena de azar y situaciones inesperadas. Desde el trabajo en sector el alto, estas incertidumbres fueron observadas a partir del ausentismo y la irregularidad en la participación de los vecinos, las cuales fueron convertidas en oportunidades, en el caso del grupo operativo, donde se trasladó el trabajo a domicilios particulares y se presentó como un desafío para la JJVV para realizar mayores esfuerzos en integrar más vecinos en las asambleas.

De igual manera, el quehacer del PC en Chile se puede ver influenciado por ciertas características del sistema sociopolítico del país. En relación a los sistemas socio políticos actuales, Bauman (2008) realiza una aproximación a cómo la modernidad tardía y la globalización convierte a las personas en "consumidores ideales", sujetos alienados que buscan satisfacción inmediata sin la capacidad de encontrar descanso si en sus vidas no existe necesidad. Es adecuado destacar que esta condición no está relacionada con las carencias básicas que puedan existir en el núcleo familiar o la comunidad, más bien forma parte de necesidades que tienen un carácter inmediato y que pueden ser satisfechas de forma rápida para dar cabida a nuevas necesidades efímeras por satisfacer. La condición de alienación entonces, es visibilizada en la comunidad a través de la poca capacidad de ocupación en relación a las necesidades sentidas, a causa de que no han podido ser problematizadas, por la condición individualizante de cada uno de sus miembros. Estas necesidades se reflejan en el barrio en, por ejemplo, el descuido de las personas mayores por parte de la sociedad, donde se detectaron problemáticas como la vulneración de derecho, el arrebato de patrimonio y el abandono; condiciones que los miembros del grupo operativo consideraban indignantes.

Igualmente, la verticalidad, es un componente que puede influir negativamente en el rol del PC, ligado con formas de intervención de carácter paternalistas (Finkler, Caetano y Sousa, 2015). Paternalismo que se caracteriza por entregar beneficios a las personas desde una visión carencial. Es así que al ver a los territorios como carentes de recursos para salir adelante se levantan intervenciones desde afuera. Al contrastar esto con la experiencia investigativa, se constató la utilización y aplicación de herramientas que venían diseñadas, ejecutadas y evaluadas por los tesistas de manera aislada, lo que pudo mermar la participación comunitaria. El dejar de lado a la comunidad invisibiliza el saber común de las personas, lo que puede invalidar su potencial transformador, instrumentalizando las relaciones de trabajo. A pesar de que se informó de la aplicación de estas herramientas (como la observación participante, las entrevistas y el diario de campo) no se logró integrar a las participantes del grupo operativo, lo que puede limitar las formas de evaluación y las perspectivas sobre la información recopilada. De esto se comprende que, a pesar de los intentos por buscar la participación en el presente proceso investigativo, también se incurrió en acciones verticales, al realizar planificación pre- interventivas fuera del contexto comunitario, bajo el "modus operandis" de la institucionalidad.

También, algunos lineamientos institucionales en la presente experiencia tuvieron un impacto similar al que han tenido algunas políticas públicas en el rol del PC. Relacionado a esto, Astete y Vaccari (2017, p.37) exponen que "las políticas sociales propuestas desde los modelos neoliberales obran desde una lógica subsidiaria que enfatizan emprendimientos económicos individuales, mermando la identidad social de algunas prácticas comunitarias, reproducción de la memoria social, acción política y solidaridad horizontal". Esto se puede extrapolar en cierta 
medida a operatividades desde otras instituciones, en este caso la academia, donde algunas consecuencias, por sus formas de funcionamiento, podría provocar rupturas con las posturas de trabajo para el Psicólogo/a Comunitario/a, siendo algunas de estas consecuencias el paternalismo y el individualismo. Si bien, la teoría expuesta no concuerda preferentemente con las interpretaciones de los hallazgos en el campo, donde los lineamientos institucionales no operaban desde un emprendimiento económico, aun así, se puede asociar con algunas limitaciones que se produjeron para la labor del PC, debido a ciertas presiones "desde arriba", que pudieron originar tensiones en el resguardo de algunas prácticas en lo comunitario.

Por otro lado, algunos componentes podrían influir positivamente en el rol del PC, siendo uno de estos la problematización. Para Hernández (2017) la problematización se presenta como una "habilidad científica básica que consiste en generar problemas en torno a una realidad específica y en someter a serios cuestionamientos toda información o circunstancia que se presenta" (p.59). Estas formas de cuestionamientos fueron visualizadas en el barrio desde el actuar de algunos miembros de la comunidad y los tesistas; a su vez, fue comprendida como una habilidad metodológica para la/el PC, permitiéndole catalizar diversas temáticas dentro de los grupos a fin de facilitar las discusiones hacia procesos reflexivos.

Según lo anterior, la reflexión crítica implicaría la evaluación de varias aristas en un problema para alcanzar una respuesta. En la JJVV, este concepto nació principalmente de discusiones grupales, donde cada vecino aportaba su perspectiva respecto a una problemática y en conjunto se debatían los contenidos de sus opiniones. De esta manera, los tesistas, en su rol de psicólogos comunitarios, podían aportar opiniones con fundamentos teóricos de la disciplina que fueran enriquecedoras de las reflexiones y con estos discursos intentar otorgarle un contexto político a cada debate.

Otro componente relevante a destacar como influyente en el rol del PC es la concientización. Desde el pensamiento Freiriano, la concientización se refiere a la "toma de consciencia de manera crítica, al investigar e interrogar la realidad (contestar y no simplemente aceptar los hechos), pero también tiene un carácter práctico, es comprensión e intervención en la realidad, en la historia" (Oliveira, Braga, y Avelino, 2018, p.77). Es así como la conciencia le otorga un sentido a una idea, que es crítica a partir del principio de cuestionar un aspecto de la realidad (reflexión crítica) y se hace práctica al intervenirla, en busca de nuevas nociones o de la transformación. En el "Sector el Alto", la toma de conciencia de aspectos propios, desde los participantes de la comunidad, funcionó como un recurso fundamental para el PC visualizado como un proceso complejo posterior a la problematización y la reflexión crítica.

Finalmente, relacionado con la movilización hacia la participación, Montero (2010) realiza una asociación de la entropía con este término. La autora problematiza la entropía constante que forma parte de la dinámica cotidiana en los espacios comunitarios. Esta realidad determina la posibilidad de logro de las actividades que se estén realizando, junto con los afectos, las condiciones tanto personales como familiares, entre otros muchos factores comprometidos que facilitan o merman la participación dentro de los grupos, como también en la presencia de los y las vecinas en las reuniones de la JJVV. Es así como en el presente estudio se observa la creación de un "nosotros" que es mencionado por parte de las participantes cuál binomio necesario para la co-construcción de significados y sentidos compartidos que posibilitaron avanzar en relaciones de calidad, imprescindibles en la creación de un grupo que se caracterice por el compromiso y disposición hacia la participación de cada persona. Esto se registró en la valoración que las participantes hacían del otro/a, siendo capaces de sentir tanto la presencia 
como la ausencia y las condiciones materiales y/o contextuales que los pudieran afectar; de esta forma las personas se comprometieron desde la alteridad y unificaron así la participación a través de la horizontalidad, abrazaron las contradicciones y diferencias de realidad grupales.

En tercer lugar, respecto a algunas competencias que debería tener el PC, las habilidades metodológicas, según Cabrera, Larraín, Moretti, Arteaga y Energici (2010), son aquellas competencias en las cuales el psicólogo debiera estar instruido de forma específica. De las competencias se destacan el análisis crítico; la aplicación de conocimientos teóricos a la práctica; manejo de conocimientos teóricos de psicología y otras áreas; comprensión e interpretación (incluye generación de hipótesis); diseño de propuestas de intervención; detección de necesidades; y gestión de proyectos. En la experiencia del presente estudio, sólo la problematización y la reflexión crítica fueron contempladas como habilidades metodológicas, debido a su pertinencia en la observación de la realidad y al estar relacionadas con algunos conocimientos teóricos de la psicología. Probablemente, el alcance del procedimiento de investigación no permitió ahondar en otros aspectos, como la gestión de proyectos y el diseño de intervenciones, quedando estos aspectos no considerados dentro de los resultados de la presente investigación.

Así mismo, las habilidades sociales transformadoras desde el pensamiento de Barroeta (citado en Olivares, 2014) se asocian con "la capacidad de los y las profesionales de generar lecturas críticas -a través de la comprensión de las estructuras de poder en las cuales están situadas sus intervenciones- para transformar las construcciones en el territorio hacia acciones proactivas que tengan como fin el cambio social" (p.5). Por consiguiente, es posible afirmar, según lo realizado por los tesistas, que las características que componen las habilidades sociales transformadoras en la presente investigación son elementos de gran importancia para el posicionamiento del PC en las comunidades. Esto ocurre porque, al utilizar estas habilidades, el PC puede generar críticas hacia algunas formas de abordaje de lo comunitario, permitiendo movilizar a los miembros de la comunidad, siempre en la búsqueda de la transformación social. En la JJVV, se valoró este actuar tras realizado el levantamiento de necesidades, agradeciendo el apoyo a los tesistas por su actitud comunitaria.

Finalmente, el actuar ético del PC, según los planteamientos de Montero (citada en Olivares et al., 2018) tiene relación con "valorar la construcción de conocimientos desde una perspectiva histórica, a través de la creación de una relación dialógica entre expertos y comunidad" (p.3). En este sentido la ruptura sujeto-objeto, desde el prisma que fue observada la comunidad, podría haber facilitado el trabajo de carácter participativo en el campo. A la vez el actuar ético sirvió de guía a los tesistas para detectar ciertas prácticas verticales.

\section{CONCLUSIONES}

\section{El rol del psicólogo/a comunitario/a como un facilitador de recursos intangibles}

Desde los principios epistemológicos de la Psicología comunitaria, el/la Psicólogo/a comunitario/a desempeña un papel de facilitador/a de recursos dentro de las comunidades; este rol facilitador se relaciona con una amplia gama de recursos intangibles. Estos elementos se asocian con lograr abogar por la propia participación activa como también la de los miembros de las comunidades, e igualmente favorecer procesos movilizadores de las conciencias en la comunidad a partir de la problematización, la reflexión crítica y la concientización, para así lograr el cumplimiento de una labor según las bases de la psicología comunitaria. 
De igual manera, como hallazgos inesperados cabe mencionar que los investigadores también pudieron incurrir en prácticas verticales desde otras instituciones ajenas a las PP, al intentar realizar una diferenciación teórica-práctica de las tensiones que surgen desde el trabajo en instituciones públicas. Estas prácticas pueden ser interpretadas como productos de algunas diferencias entre los tiempos comunitarios y los tiempos institucionales, donde el/la PC queda supeditado a responder a las exigencias de un lado por sobre el otro, dependiendo de las circunstancias y de sus propias capacidades de afrontamiento. Igualmente, otro hallazgo inesperado tiene que ver con la gama de amenazas hacia el trabajo del PC, que se relacionaría con aspectos socio-políticos que influyen en la cotidianidad de las personas, pero que también se pueden presentar como oportunidades para la intervención.

Además de lo anterior, también hay que considerar que los hallazgos encontrados pueden ser de relevancia teórica/práctica para otros estudios relacionados con la reflexión sobre la praxis del PC en el contexto Chileno, ya que permiten tomar en cuenta aquellos aspectos mencionados como amenazas y limitaciones para la labor del PC y de esta manera intentar no caer en prácticas no deseadas. También, a modo de recomendación para futuras experiencias, serviría el identificar oportunamente aquellas dinámicas que mermen lo participativo, procurando desarrollar aquellas acciones que sí consideran a los miembros de las comunidades, para de esta forma lograr realizar investigaciones en resguardo de los valores de la Psicología Comunitaria. Igualmente, si bien, se logró responder en buena medida a las preguntas de investigación y los objetivos fueron cumplidos con la comunidad, se presenta como una limitación importante de la investigación el no lograr la participación total de los vecinos en la observación del rol del Psicólogo Comunitario.

Por último, aún queda por profundizar la problemática del verticalismo en las prácticas del PC y así determinar si estas se originan en su totalidad desde las instituciones o políticas públicas, o si también están relacionadas con las capacidades del interventor de mediar entre estas y la comunidad, permitiendo de esta forma develar si es necesario centrar los esfuerzos de algunos/as psicólogos/as comunitarios/as en transformar ciertas políticas públicas desde las bases comunitarias, o centrar este cambio en su propia manera de operacionalizar sus acciones.

\section{REFERENCIAS BIBLIOGRÁFICAS}

Alfaro, J. y Barroeta, H. (2007). Trayectoria de la Psicología Comunitaria en Chile.Prácticas y conceptos. Valparaíso, Chile: Universidad de Valparaíso.

Alfaro, J., y Martín, M. (2015). Proceso y oportunidades de la transferencia del conocimiento desde la psicología comunitaria a las políticas públicas. Universitas Psychologica, 14(4), 1347-1358.

Almeida, E., y Sánchez, M. E. (2014). Comunidad: interacción, conflicto y utopía. México: Universidad Iberoamericana, ITESO y BUAP.

Ander-Egg, E. (1981). Metodología y práctica de la Animación Sociocultural. Madrid, España: Instituto de Ciencias Sociales Aplicada.

Arias, A. (2015). Communitarian Psychology: Posibilities and capacities. Psicogente, 18(34), 260-266.

Astete, M., y Vaccari, P. (2017). Políticas públicas y subjetividades: Lógicas en disputa en la implementación de programas sociales en la comuna de Lota, Chile. Psicoperspectivas, 16(1), 31-41.

Balcazar, F., Montero, M., Newbrough, J. R., y Porbén, M. (2003). Modelos de Psicología Comunitaria para la Promoción de la Salud y Prevención de Enfermedades en las Américas. Revista Interamericana de Psicología, 37(1), 181-188.

Bauman, Z. (2008). La Globalización: Consecuencias Humanas. Buenos Aires, Argentina: Fondo de Cultura Económica.

Batlle, R. (2013). ¿Qué es el Aprendizaje-Servicio y por qué nos interesa? Monográficos Escuela, (nov), 45. Recuperado de: http://roserbatlle.net/wp-content/uploads/2014/10/monografico-aps-periodicoescuela-2013.pdf

Bénard, S. (Ed.). (2016). Teoría Fundamentada: Una metodología cualitativa. Recuperado de: https://www.uaa.mx/direcciones/dgdv/editorial/docs/ve_teoria_fundamentada.pdf

Cabrera, P., Larraín, A., Moretti, R., Arteaga, M., y Energici, $\bar{M}$. (2010). La formación en psicología desde una perspectiva de competencias. Una contribución para el mejoramiento de la formación universitaria en chile. Calidad en la Educación, (33), 183- 221. 
Carrasco, C., Cerda, G., Baltar, M. J., Zamora, C., y Castillo, Á. (2016). Tensiones de un centro comunitario: análisis desde la psicología comunitaria en Chile. Revista de psicología (Santiago), 25(1), 1-22.

Dussel, E. D. (2017). Filosofías del Sur. Descolonización y transmodernidad. Ciudad de México, México: Ediciones Akal.

Finkler, M., Caetano, J. C, y Sousa, F. R. (2015). Professional ethics training ethics: teacher-student paternalism of professor-student relationship in the context of the clinical relationship. EIDON, (44), 43-45.

García, J. (2011). Aprendiendo a hacer escuelas. Las complejas y dinámicas relaciones entre bachilleratos populares y el Estado. (Tesis de maestría). UBA, Buenos Aires, Argentina.

García, J., Priego, O. y Rebollo, J. (2018). Globalización y crisis como factores Condicionantes de pobreza, desigualdad y hambre en América Latina. En S. De la Vega y C. Rodríguez. (Ed.), Desigualdad Regional, Pobreza y Migración. (pp. 666-679). Coyoacán, México: UNAM.

García, R. (2017). Edgar Morin y Los 7 Saberes: Hacia un esquema interpretativo. Revista Electrónica Sinergias Educativas, 1(1), 73-84.

Graterol, G. (Septiembre de 2010). Educaciòn para la ciudadanía. La educación comunitaria y la participación ciudadana: debates actuales de la transformación social en Iberoamérica. En M. I. Vollmer (Presidenta), Metas 2021: La educación que queremos para la generación de los Bicentenarios. Conferencia llevada a cabo en el Congreso Iberoamericano de Educación (Metas 2021) de la OEI, Buenos Aires, Argentina.

Hernández, A. (2017). Procesos metodológicos para desarrollar habilidades investigativas en los alumnos de la Escuela Profesional de Psicología de la Universidad "Señor de Sipán" de Chiclayo 2016. (Tesis de Maestría). Universidad Nacional Pedro Ruiz Gallo, Lambayeque, Perú.

Ketterer, L. M., Mayorga, C., Carrasco, M., Soto, A., Tragolaf, A., Nitrihual, L., y Del Valle, C. (2017). Modelo participativo para el abordaje de la violencia contra las mujeres en La Araucanía, Chile. Revista panamericana de salud pública, (41), 1-4.

Leones, E. (2018). Aproximación a los principios éticos de la liberación del sujeto latinoamericano en el pensamiento de Enrique Dussel. Amauta, 16(31), 49-70.

Malluk, A. L. y D'Luyz, C. A. (2016). Evaluación del Programa Reciclando Ando, en el marco de la responsabilidad social universitaria. Estudio de caso. Universidad Bolivariana, 5(9), 45-72.

Martín-Baró, I. (1990). Acción e ideología. San Salvador, El Salvador: UCA.

Martín-Baró, I. (2017). Crítica e libertação na psicologia: Estudos psicossociais. Petrópolis, Brasil: Vozes.

Matos, R. (2006). La práctica de la reflexión durante el aprendizaje de un instrumento musical. Revista de investigación, 30(59), 65-88.

Montero, M. (2003). Teoría y práctica de la Psicología Comunitaria. La tensión entre comunidad y sociedad. Buenos Aires, Argentina: Paidós.

Montero, M. (2010). Crítica, autocrítica y construcción de teoría en la psicología social latinoamericana. Rev. Colombiana de Psicología, 19(2), 177-191.

Gough (Ed.), The Palgrave Handbook of Critical Social Psychology, (pp. 147-161). Londres, Inglaterra: Palgrave Macmillan.

Olguín, M. I., Urzúa, L., Vargas, C. y Vidal, A. (2016). Percepciones de las familias en cuanto a su participación en el proceso de rehabilitación psicosocial de usuarios de un Centro Diurno en la Región de Valparaíso. (Trabajo de pregrado). Pontificia Universidad Católica de Valparaíso, Valparaíso, Chile.

Olivares, B. (2014). Políticas Públicas y Psicología Comunitaria: Un estudio de caso sobre los programas comunitarios en la política pública de infancia. (Tesis de Maestría). Universidad de Chile, Santiago, Chile.

Olivares, B., Reyes, M. I., Berroeta, H. y Winkler, M. I. (2016). La Formación Universitaria en la Psicología Comunitaria Chilena de Hoy: ¿Un Lugar Subalterno? Psykhe, 25(2), 1- 12.

Olivares, B., Winkler, M. I., Reyes, M. I., Berroeta, H. y Montero, M. (2018). ¿Y si pensamos la comunidad con derechos? Psicología Comunitaria, derechos y políticas públicas. Una relación compleja. Universitas Psychologica, 17(2), 1-13.

Oliveira, C., Braga, F., y Avelino, C. (2018). Las Contribuciones del Concepto de Alfabetización de Paulo Freire Para Los Estudios Sobre La Educación En Derechos Humanos. Paulo Freire. Revista de Pedagogía Crítica,(19),65-88.

Posadas, P., y Godino, J. (2017). Reflexión sobre la práctica docente como estrategia formativa para desarrollar el conocimiento didáctico-matemático. Didacticae, (1), 77-96.

Puig, J. M., Batlle, R., Bosch, C., y Palos, J. (2007). Aprendizaje servicio. Educar para la ciudadanía. Madrid: Octaedro.

Restrepo, C. y González, H. (2018). El Destierro. Una lectura del desplazamiento forzado desde la Educación Popular -Subjetividades y Ciudadanía-. (Trabajo de pregrado). Universidad del Valle, Cali, Colombia.

Reyes, M. I., Olivares, B., Barroeta, H. y Winkler, M. I. (2015). Del discurso a las prácticas: Políticas sociales y psicología comunitaria en Chile. Polis, 14(42), 387-413.

Tapia, N. (2018). Guía para desarrollar proyectos de aprendizaje-servicio solidario -edición Perú-. Buenos Aires: Centro Latinoamericano de Aprendizaje y Servicio Solidario (CLAYSS). Recuperado de http://www.clayss.org.ar/04_publicaciones/Manual_Peru_para_web.pdf 